\title{
Calcium Phosphates and Angiogenesis: Implications and Advances for Bone Regeneration
}

Citation for published version (APA):

Malhotra, A., \& Habibovic, P. (2016). Calcium Phosphates and Angiogenesis: Implications and Advances for Bone Regeneration. Trends in Biotechnology, 34(12), 983-992.

https://doi.org/10.1016/j.tibtech.2016.07.005

Document status and date:

Published: 01/12/2016

DOI:

10.1016/j.tibtech.2016.07.005

Document Version:

Publisher's PDF, also known as Version of record

Document license:

Taverne

Please check the document version of this publication:

- A submitted manuscript is the version of the article upon submission and before peer-review. There can be important differences between the submitted version and the official published version of record.

People interested in the research are advised to contact the author for the final version of the publication, or visit the DOI to the publisher's website.

- The final author version and the galley proof are versions of the publication after peer review.

- The final published version features the final layout of the paper including the volume, issue and page numbers.

Link to publication

\footnotetext{
General rights rights.

- You may freely distribute the URL identifying the publication in the public portal. please follow below link for the End User Agreement:

www.umlib.nl/taverne-license

Take down policy

If you believe that this document breaches copyright please contact us at:

repository@maastrichtuniversity.nl

providing details and we will investigate your claim.
}

Copyright and moral rights for the publications made accessible in the public portal are retained by the authors and/or other copyright owners and it is a condition of accessing publications that users recognise and abide by the legal requirements associated with these

- Users may download and print one copy of any publication from the public portal for the purpose of private study or research.

- You may not further distribute the material or use it for any profit-making activity or commercial gain

If the publication is distributed under the terms of Article $25 \mathrm{fa}$ of the Dutch Copyright Act, indicated by the "Taverne" license above, 


\section{Review}

Calcium Phosphates and

Angiogenesis: Implications and Advances for Bone Regeneration

\author{
Angad Malhotra ${ }^{1}$ and Pamela Habibovic ${ }^{1, *}$
}

Calcium phosphates (CaPs) are among the most utilized synthetic biomaterials for bone regeneration, largely owing to their established osteoconductive and osteoinductive properties. While angiogenesis is a crucial prerequisite to bone formation, research and applications for CaPs have not appreciated its crucial role. This review discusses how CaPs influence angiogenesis, and highlights promising strategies that address this topic. The objective is to draw attention to the gap in the literature and to highlight the importance of angiogenesis in $\mathrm{CaP}$ research, development, and use.

\section{Why Consider Angiogenesis in CaP-Augmented Bone Regeneration?}

Traditionally, three components guide bone tissue engineering (see Glossary): osteoconductivity, osteoinductivity, and osseointegration [1,2]. With a long history of development, CaPs fulfill two of these criteria: they are commercially available as osteoconductive bone-graft substitutes and have demonstrated osteoinductive potential [3-5]. One of the most relevant features of CaPs is their chemical similarity to the inorganic phase of bone matrix [6], providing a rationale for their use. Over decades, extensive research on CaP-based materials has aimed to improve their potential for osteogenesis-related processes; however, these efforts have not focused on how CaPs influence angiogenesis, despite it being a crucial process for bone formation.

Vascularization is vital for bone defects to heal, which in turn facilitates the development of actual osteoid deposition and matrix development within normal bone healing. Importantly, a coupling link exists between the angiogenic and osteogenic cells and signals, underscoring the importance of these interactions (Box 1). In practice, there is a greater need for vascularization at sites where bone graft substitutes are used because they are larger, more complex environments, and require more control in tissue formation and development. Tissue engineering approaches generally employ a classical three-way focus on osteoconduction, osteoinduction, and osteogenesis. By contrast, the more clinically relevant so-called diamond concept acknowledges the complexity of the bone niche by considering the vasculature and the mechanical environment [7]. This concept, together with deeper insights into the coupling of healing mechanisms, highlights the oversight and misalignment of $\mathrm{CaP}$ research and development, and supports a greater consideration for angiogenesis in related $\mathrm{CaP}$ research. In developing bone-graft substitutes that take into account the importance of angiogenesis, a wealth of information can be obtained from other fields (Box 2).

Bone healing is a complex process that consists of various overlapping phases with many cellular and molecular processes or requirements [8]. Complications in this process, such as

\section{Trends}

There is increasing demand for synthetic alternatives to a patient's own material in regenerative strategies for damaged or diseased tissues and organs. In bone regeneration, CaPs are promising candidates.

While traditional $\mathrm{CaP}$ designs were focused on the initiation and guidance of new bone formation, the next generation of $\mathrm{CaP}$ materials needs to better address the complexity of the bonehealing environment.

Successful CaP bone-graft substitutes for clinically relevant bone defects must be able to establish and maintain proper vasculature.

Instead of adding cells or growth factors to establish both angiogenesis and bone growth, the focus is now on inorganic or small molecules, and on the improved design of CaPs. These priorities aim to enhance the clinical performance and affordability of $\mathrm{CaPs}$.

${ }^{1}$ MERLN Institute for Technologyinspired Regenerative Medicine, Maastricht University, The Netherlands

${ }^{*}$ Correspondence:.

p.habibovic@maastrichtuniversity.nl (P. Habibovic). 


\section{Box 1. Cells and Signals Relevant to Blood Vessels Within Bone}

Blood vessels transport oxygen, nutrients, and waste, and they also act as a passageway for immune system components, cell-signaling molecules, and cells [79-81]. Endothelial cells (ECs) form the capillary tubes where component transfer occurs [82], and these connect the circulatory loop of arteries and veins. Vascular smooth muscle cells (VSMCs) and pericytes form the arterial and venous vessels, and regulate EC proliferation via cell contact and paracrine signaling $[78,83]$. EC proliferation and lateral migration are the major source of new ECs, although the progenitors are predominantly bone marrow-derived [84].

Many signaling molecules are involved in angiogenesis, of which vascular endothelial growth factor (VEGF) and basic fibroblast growth factor (bFGF) are the most prominent. VEGF and bFGF exert chemotactic and mitogenic functions by binding to their associated receptors on ECs, inducing the release of matrix metalloproteinases (MMP) that degrade components of the extracellular matrix. Proliferating and migrating ECs sprout into this newly formed space, forming new blood vessels, while tissue inhibitors of metalloproteinases (TIMPs) regulate MMP activity [85].

Sprouting ECs can be differentiated by their position and function into tip and stalk ECs. Both tip and stalk ECs are controlled by Notch signaling pathways, with tip cells expressing Notch ligand DLL4 and stalk cells having high Notch expression. Tip cells sprout vessels according to optimal oxygen delivery, while stalk cells form the basement membrane components that form the lumen of the vessels. Complex interactions exist between VEGF receptors, Notch and DLL4 signaling, and feedback loops [79]. Recently, a specific subpopulation of ECs was described, defined as type H ECs, which more strongly express CD31 and endomucin cell-surface markers compared to type L ECs. Osteoprogenitors, expressing Runx2 and Osterix, indicative of osteoblastic precursors, were located directly around type $\mathrm{H}$ vessels [80]. This type $\mathrm{H}$ subpopulation also secretes noggin, a bone morphogenetic protein antagonist, but sustainer of osteoblasts. These osteoblasts in turn secrete VEGF, coupling angiogenesis and osteogenesis mechanisms [81]. Such bone microvessel type $\mathrm{H}$ ECs are regulated by $\mathrm{HIF-1} \propto$ and DLL4-Notch signaling mechanisms [80], and are an interesting differentiator of EC subtypes relevant to bone regeneration and targets for CaPs.

delayed healings and non-unions, are often attributed to a failure in restoring vasculature, and not to a lack of osteogenic potential [9]. Bone-graft substitutes are often needed under circumstances of increased risk, but without the prerequisite blood supply, the healing environment struggles to even reach the phase when osteogenesis is required. Further, $\mathrm{CaP}$ implantation from time-zero aligns with the phase related to hematoma formation and inflammation; although osteogenic differentiation is not yet a concern, it should not be neglected.

To understand bone formation at a systems level, researchers must consider endothelial cells (ECS), osteoblasts, and their innate linkage. Preosteoblasts are recruited and maintained by interaction with ECs, ensuring suitable migration to the place of need [10]. When considering $\mathrm{CaP}$ use within a bone defect, a functioning capillary network provides a migration pathway for endogenous cells [11], and this pathway is crucial for delivering osteoprogenitor cells to the often otherwise cell-free CaP scaffold. Therefore, the coupling of osteogenesis and angiogenesis is

\section{Box 2. Lessons Learned from the Oncology Field}

Considerable knowledge about angiogenesis has been established within tumor progression and metastasis research, where limiting angiogenesis is crucial in suppressing tumor development. Within this realm, angiogenesis is considered to be a highly regulated process, switching on for days, followed by inhibition, as evident with angiogenic phenotype activation of tumor cells in tumor development and growth [86]. Despite the cell densities, ECs are most often quiescent, with only $0.01 \%$ dividing at any time [87]. In relation to bone healing, there is great potential in activating proliferation temporally, capturing the angiogenic switching window of the bone-healing environment at the early stages of healing, and limiting any stagnant cell populations. For tumor control, antiangiogenic agents are deployed, such as VEGF inhibitors, whose goal is to limit EC proliferation to prevent vessel growth, to promote EC death, and to suppress the mobilization of progenitor ECs from the bone marrow [82]. Hence, stimulating vessel regeneration for bone healing requires suitable signaling to induce EC migration and proliferation, and focus on these mechanisms is a justified aim. Another interesting approach is the inhibition of specific signaling pathways. While VEGF signaling is known to be essential for bone healing, halting angiogenesis in disease and disorders using VEGF inhibition alone is considered insufficient owing to associated upregulation of complementary factors [82]. This suggests that single-mechanism strategies may be limited because their effects may be compensated by the downregulation of other processes. In addition, the importance of a single angiogenic factor does not imply that supraphysiological levels will actually be beneficial.

\section{Glossary}

Angiogenesis: the process and stimulation of new blood vessel formation via sprouting from existing blood vessels.

Bioinorganics: inorganic elements that are often naturally occurring within the body in trace quantities, and which can stimulate and influence a wide range of cellular processes.

Bone tissue engineering: the three components traditionally considered to be essential are osteoconductive biomaterials such as calcium phosphates (CaPs), osteoinductive signals such as growth factors, and bone-forming cells such osteogenic cells and mesenchymal stem cells.

\section{Delayed healings and non-unions:} the extensive formation of fibrocartilage across a bone defect site leads to healing complications, which may be more likely with increasing soft tissue and vascular damage. No consensus exists in how to classify these events, although it is sometimes considered to be $>3$ months for delayed healings and $>9$ months for non-unions.

Diamond concept: a broad concept in bone healing that builds on the three components of bone tissue engineering to include the mechanical environment and the vasculature, as well as considering the biological variations of the host system.

Osteogenic cells: cells that are related to bone formation, such as osteoprogenitors, preosteoblasts, bone-lining cells, and osteoblasts.

Osteoconduction: the ability of the material to facilitate the attachment of osteoprogenitor cells, necessary for bone formation on or throughout the material.

Osteoinduction: the stimulation of undifferentiated and multipotent cells towards osteoblasts capable of depositing bone matrix.

Proangiogenic factors: any substance capable of stimulating angiogenesis, of which bFGF and VEGF are most commonly considered, although many cofactors and crosstalk can exist. 

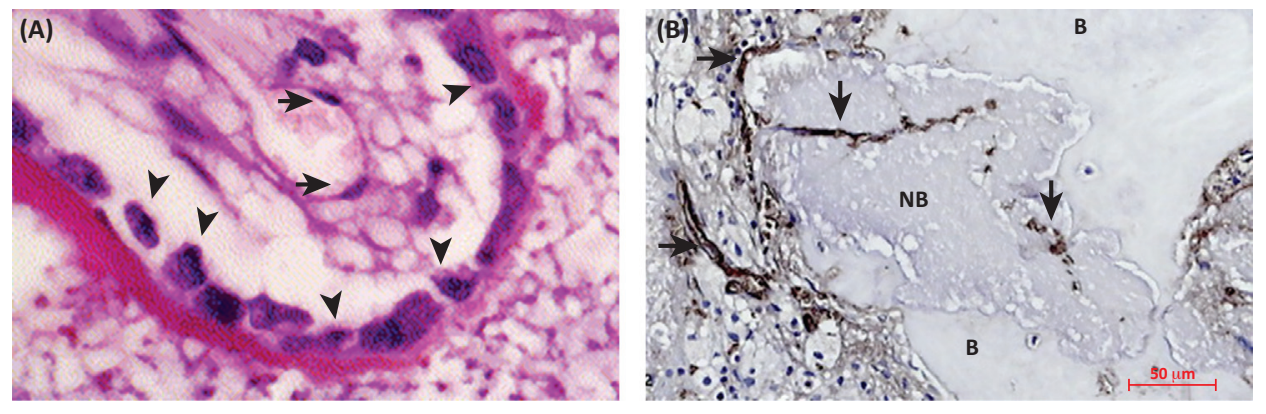

Trends in Biotechnology

Figure 1. (A) Osteoblasts (Triangles) Line the Surface of a Pore of the Calcium Phosphate (CaP) with Endothelial Cells (Arrows) on a Hematoxylin/Eosin/Safranin Stained Section ( $\times 1000)$. Pore dimensions can influence vessel formation and blood supply to the osteoblasts, and thus bone formation. Reproduced, with permission, from Henno et al. [88]. (B) Capillaries (arrows), highlighted with von Willebrand factor immunostaining, demonstrate the penetration of vessels into a CaP-based product embedded in a $\mathrm{SiO}_{2}\left(\mathrm{NovaBone}^{\circledR}, \mathrm{NB}\right)$, surrounded by new bone (B). Reproduced, with permission, from Götz, et al. [12].

essential in the bone-healing environment [12] (Figure 1). Furthermore, the close connection of blood vessels and bone cells maintains skeletal integrity, an underappreciated relationship in $\mathrm{CaP}$ development. This review discusses current and potential strategies to improve the angiogenic response when $\mathrm{CaPs}$ are used for the augmentation of bone defects. Such information could help to shape a new generation of $\mathrm{CaP}$ bone-graft substitutes that more effectively and relevantly address the needs of the regenerating bone system.

\section{What are the Roles and Relevant Effects of CaP Material?}

The physiological importance of both calcium [13-15] and phosphate [16-18] has been well described. Calcium and phosphate are regulated throughout the body via a homeostatic pathway, the vasculature. While calcium and phosphate are crucial for homeostasis, increased levels have been negatively associated with vascular calcifications [19,20], vascular smooth muscle cell (VSMC) apoptosis [19], and EC oxidative stress [21] and apoptosis [22]. CaP materials degrade via solution-, protein-, and cell-mediated mechanisms, resulting in the ionic transfer of calcium and inorganic phosphate from the solid to the liquid phase [23]. Further research will be necessary to determine whether a local excess of CaP degradation products aids or negatively influences revascularization within the bone-healing environment as well as to elucidate possible systemic effects.

In addition to the chemical changes induced by $\mathrm{CaP}$ degradation, the mechanical and physical aspects of introducing CaPs also need to be considered. Both ECs and VSMCs are known to respond to mechanical stretching that induces gene expression and protein synthesis through mechanoreceptors such as stretch-activated channels, integrin proteins, and CD31 [24]; whether they also respond to strains imposed on $\mathrm{CaP}$ remains to be determined. Computational modeling has described the effects of CaP strain on angiogenesis [25], but CaP-induced changes on tissue stiffness have not been deeply investigated in relation to angiogenesis. Physical features of $\mathrm{CaP}$ materials, such as porosity, also influence new vessel formation: interconnected pore sizes have been reported to be beneficial in the range of 100-150 $\mu \mathrm{m}$ $[26,27]$ or even larger depending on the vessel diameter [28], while macropores in the range of 300-400 $\mu \mathrm{m}$ have been often reported to be favorable [29]. Furthermore, the specific phase of the CaP can also impact on angiogenesis [30]. Importantly, these physical features relate to the mechanical properties and degradation rate of the material. Because the load-bearing capabilities of CaPs are often mentioned as a limitation [31], the balance between multiple mechanical and physical properties is an important consideration for future $\mathrm{CaP}$ design. 


\section{Angiogenic Possibilities with CaPs}

Many strategies have been investigated for adding various biological and synthetic agents with cell-signaling capabilities, including soaking, encapsulation, or covalent bonding of growth factors [32]. These strategies for CaPs have been previously reviewed in an osteogenic context [6]. We focus here on methods to specifically address angiogenesis with CaP use.

\section{Biological Options}

Autologous growth factors, those sequestered from one's own body, contain proangiogenic factors such as vascular endothelial growth factor (VEGF) and basic fibroblast growth factor (bFGF). Autologous platelet-rich plasma combined with CaP granules was shown to improve overall bone healing in vivo [33]. Similarly, fibrin glue added to a CaP cement was reported to improve revascularization specifically, and here a 1:1 ratio was considered most efficacious when implanted intramuscularly in rabbits [34]. Fundamentally, this approach recreates normal bone healing, where platelet degranulation during hematoma formation provides the environment with a variety of growth factors. However, an issue with this approach is the uncontrolled delivery of unknown concentrations of growth factors, making reproducibility a concern. Recombinant growth factors and cell-signaling molecules provide an accessible alternative, while providing greater dose control than autologous methods. Recombinant proteins, such as VEGF, are a logical approach to improve vasculature. Recombinant VEGF in combination with commercially available CaP particles was shown to result in greater new bone volume in a rat calvarial bone-defect model [35] (Figure 2i).

Because VEGF expression peaks from 10-14 days post-injury [36], one issue commonly discussed is the burst release of factors used in combination with CaPs, or indeed many biomaterials. To address this concern, release over weeks is sought, rather than over hours or days. The coprecipitation of VEGF onto biphasic CaPs (BCP), consisting of hydroxyapatite and

(i)
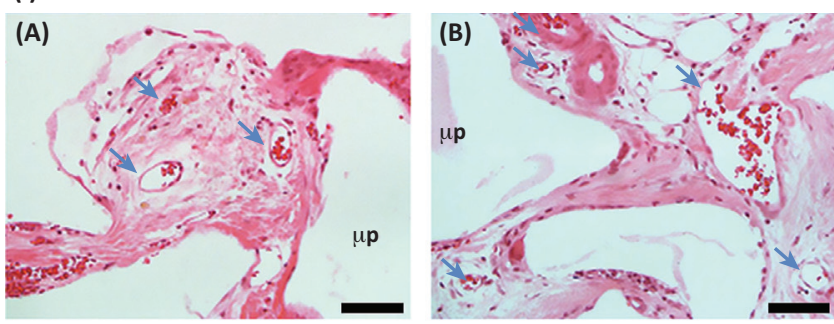

(ii)
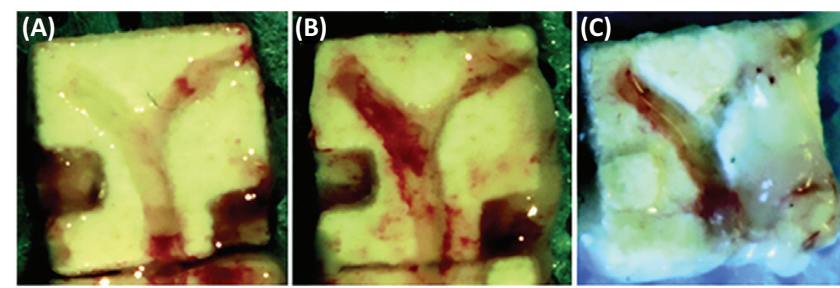

(iii)
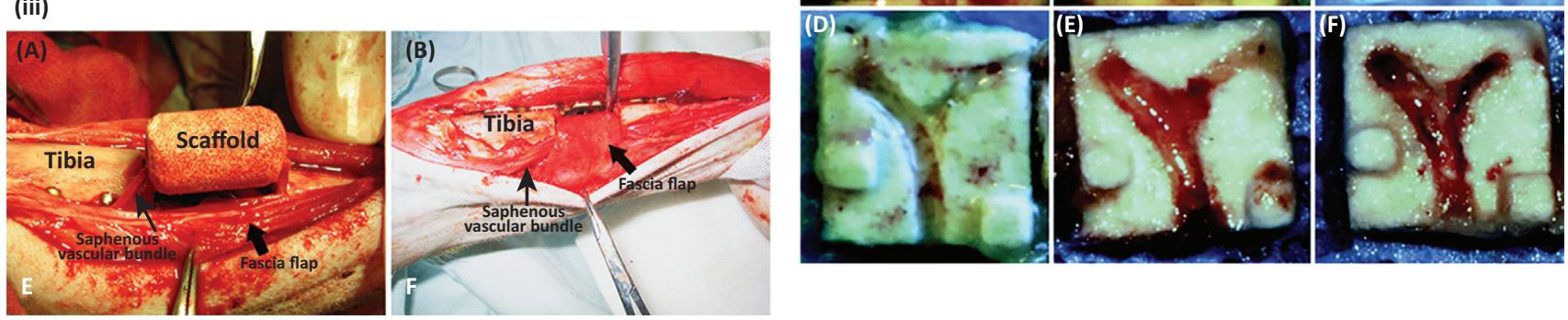

Trends in Biotechnology

Figure 2. (i) A Greater Number of Vessels (Arrows) Were Reported Within the New Bone that Formed in the Pores of Microparticles ( $\mu$ p) of VEGFLoaded CaP Bone-Graft Substitutes. Scale bars represent $50 \mu \mathrm{m}$; staining with hematoxylin/eosin; two representative samples are shown. Adapted, with permission, from Kang et al. [35]. (ii) Vessel formation was induced in a dicalcium phosphate dehydrate implant. The control (A) had limited migration, while loading with copper at $56 \mathrm{ng}(\mathrm{B}), 560 \mathrm{ng}(\mathrm{C})$, and $5600 \mathrm{ng}(\mathrm{D})$ gave dose-related improvements. When VEGF only was loaded onto implants at $0.2 \mu \mathrm{g}(\mathrm{E})$, and $2 \mu \mathrm{g}(\mathrm{F})$, vessel formation was even greater, demonstrating the beneficial effect of combining promoters of angiogenesis with CaP implants. Adapted from Barralet et al. [52]. (iii) The CaPbased scaffold was loaded with MSCs and augmented by inserting a vascular bundle into the side grooves of the scaffold and covering it with a fascia flap. Adapted, with permission, from Fan et al. [64]. 
$\beta$-tricalcium phosphate $(\beta$-TCP), was reported to benefit vascularization in cranial bone defects in mice, which was attributed to more sustained VEGF release [37], and reiterates the need to align the release kinetics with the needs of the environment. Other growth factors and combinations have also been successfully implemented with CaPs. In combination with a BCP, bFGF upregulated the expression of markers of osteogenic differentiation of mesenchymal stem cells (MSCs) in vitro [38]. In an in vivo study, the combination of bFGF, bone morphogenetic protein 2 (BMP-2), mesenchymal stem cells (MSCs), and hydroxyapatite particles was more efficacious than MSCs with hydroxyapatite for fusing spinal vertebrae in rabbits [39]. Similarly, recombinant VEGF combined with BMP-2 loaded onto a CaP scaffold was also shown to act synergistically in calvarial bone defects in nude mice, with VEGF possibly also playing a chemotactic role for the osteoprogenitors needed for BMP-2 to exert its osteoinductive effect [40]. Despite positive results with proangiogenic growth factors, angiogenic processes are often overlooked, making it difficult to elucidate the exact mechanisms and timelines without an understanding of the preceding vascularization. This insight is especially important in studies utilizing bFGF and/or VEGF. While downsides may include the costs and concerns with safety, adding proangiogenic growth factors to CaP-based biomaterials is relatively achievable and appears efficacious. However, more understanding is needed on how to combine these growth factors effectively with $\mathrm{CaPs}$, and deeper investigation is needed into in vivo endpoints to assess successful vascularization.

In addition to using growth factors, prevascularization has been considered as another promising method. In this case vessels are formed in vitro before implantation through coculturing of MSCs and ECs to form cell aggregates [41], as well as in combination with endothelial-induced MSCs within a hydrogel [42] or a collagen-based material [43]. While a promising strategy, this approach is less commonly applied to CaPs. When ECs were combined with either hydroxyapatite or $\beta$-TCP, both of the CaPs supported a microcapillary-like network of ECs upon angiogenic stimulation in vitro [44], and this demonstrated feasibility of combining ECs with CaPs. Similarly, a coculture of HUVECs and osteoblasts on CaP cement was reported to sufficiently prevascularize CaP scaffolds in vitro $[45,46]$. In another example, exogenous sonic hedgehog combined with osteoinductive CaP granules was implanted subcutaneously in mice and was reported to induce in vitro vessel formation, displaying anastomoses with the host vasculature and subsequently more mature bone formation [47]. While prevascularization in vitro is an interesting approach, the cell source and anastomoses to the host vessels remain issues to address [48]. This successful connection to the existing vasculature appears to be the greatest challenge limiting the translation of this approach, regardless of the bone-graft-substitute material.

\section{Synthetic and Manufactured Options}

While autologous and exogenous growth factors are promising, synthetic options are an encouraging alternative, especially to limit costs and safety concerns. The inorganic portion of bone is a CaP-based mineral that contains a variety of trace elements [49]. These same inorganic elements, or bioinorganics, have been introduced as additives to CaPs and were shown to be capable of stimulating many bone-related, cellular signaling mechanisms [50]. Strontium, while often considered a promoter of osteogenesis, has been linked to the increase in proangiogenic factors from possibly osteoblasts [51], indirectly promoting vascularization. Copper, also a bioinorganic, appears to have direct proangiogenic potential. Copper sulfate adsorbed onto a 3D-printed, CaP-cement scaffold was reported to improve angiogenesis over controls within the peritoneal cavity in mice, and showed even greater improvement when used in combination with exogenous VEGF [52] (Figure 2ii). Similarly, cobalt incorporated into CaP coatings has been previously suggested to have the potential to stimulate angiogenesis [53]. However, incorporation of copper and cobalt together in CaPs was shown to reduce osteoblast proliferation and alkaline phosphatase (ALP) expression in vitro [54], as well as human MSC 
osteogenic gene expression, indicating that positive gains in angiogenesis can negatively impact on osteogenic processes. Some of these losses may be recovered in vitro using combined strategies with pro-osteogenic bioinorganics [55]. In addition, the presence of such inorganic additives within the crystal structure of the $\mathrm{CaP}$ modifies the structure and, in turn, the dissolution rate of the material [23]. This changes the availability of both the added ion, and the calcium and inorganic phosphate ions, making it even more difficult to appreciate the local ion concentrations between materials. Overall, the incorporation of bioinorganics into CaPs is a relatively cheap alternative with proven potential. Future developments of bioinorganic additives should consider issues of toxicity as well as systemic accumulation and its effects.

Besides the addition of bioinorganics, combining different materials with $\mathrm{CaPs}$ has also been shown as a successful synthetic strategy to improve the process of new blood vessel formation. Using an osteoblast and EC coculture system, a polymer and ceramic composite was found to have benefits for $\mathrm{EC}$ growth and vessel-like formation in vitro. A higher polymer ratio supported early angiogenesis and, importantly, was not reported to adversely affect osteogenic processes [56]. Similarly, CaP nanoparticles at $20 \% \mathrm{w} / \mathrm{w}$ within electrospun poly(lactic acid) upregulated VEGF expression in ECs in vitro and supported vessel formation when implanted subcutaneously in mice, suggesting a positive angiogenic effect from the composite material. Further, a composite of $\mathrm{CaP}$ housed within a polymer scaffold was reported to favor angiogenesis over the polymer alone, possibly via the chemotaxis and polarization of M1 - and M2-phenotype macrophages [57]. Composite $\mathrm{CaP}$ polymers have been suggested to improve the mechanical properties and degradation kinetics [31] and, combined with potential improvements in angiogenesis, such composites may be useful overall. Refining the combinations and ratios and obtaining greater mechanistic insight will be necessary to further develop such composite materials.

A third synthetic strategy to stimulate angiogenesis involves the creation of a hypoxic environment (Box 3). This beneficial effect has been demonstrated with small-molecule hypoxia mimickers, such as phenanthroline, to induce blood vessel formation within Matrigel plugs implanted subcutaneously in mice [58]. However, few studies have used this strategy in combination with CaPs for bone-regeneration applications. Cobalt is thought to act as a hypoxia-mimicker: in combination with a mesoporous bioactive glass, a low percentage of cobalt ions demonstrated increased hypoxia-inducible factor (HIF)-1 $\propto$ in vitro [59]. However, the calcium quantities of such glass scaffolds are very different compared to CaPs. In addition, because hypoxic conditions are thought to modulate chondrogenesis via HIF activity in developing limbs [60], inducing hypoxic conditions within bone-healing environments may promote the chondrogenic differentiation of recruited MSCs rather than their osteogenic differentiation. Combination strategies with $\mathrm{CaPs}$ and hypoxia mimickers are lacking. A potential strategy for larger bone defects may be to combine CaPs, hypoxia mimickers, and either chemotactic agents or cell delivery.

Box 3. Hypoxia and Its Role

A major role of blood vessels throughout the body is to supply oxygen to cells. Normoxic conditions are within the range of 2-9\% oxygen [60]; a lack of blood vessels limits oxygen supply, inducing hypoxic conditions within the microenvironment. Blood vessels are only considered useful to cells within a maximum range of $100-200 \mu \mathrm{m}$, the oxygen diffusion limit. Interestingly, the oxygen limit is itself a strong driver for angiogenesis, in which transcription factors such as hypoxiainducible factors (HIF) regulate many pro- and antiangiogenic factors [78,89]. This hypoxic mechanism controls angiogenesis via the upregulation of VEGF-A [80]. Enhanced HIF- $1 \propto$ has been related to the increase in osteoprogenitors and subsequent bone formation [81]. During early healing, inflammatory processes encourage bFGF release from macrophages when the oxygen tension is low and lactate is high from anaerobic metabolism [90]. This release, combined with increased angiogenic activity during fibrin or foreign material phagocytosis, contributes to revascularization [86]. Even with evidence of revascularization, the time taken to achieve the appropriate vasculature and any non-uniformity in the distribution of molecules, factors, and cells can contribute to unfavorable overall conditions and outcomes [91]. Because a lack of oxygen and blood supply can result in inadequate bone healing, unaided standard hypoxia-induced mechanisms may not be successful on their own. 


\section{Surgical and In Situ Options}

In addition to the aforementioned strategies that focus on modifications and additives to $\mathrm{CaP}$ bone-graft substitutes, numerous options exist to stimulate angiogenesis during surgery or inside the body. For example, by accessing the already functioning vasculature, free flap and muscle coverage can promote bone healing. In worst-case scenarios, such as when the periosteum tissue is also compromised, applying revascularization methods are even more important [61]. Another approach is to use a degradable bone-graft substitute as a graft extender, combined with a vascularized autograft [62] as a means to deliver vascular structures to a defect site. Alternatively, utilizing the local functioning vasculature is also possible. Arteriovenous vascular bundles placed in the grooves of a nano-hydroxyapatite-based material in rats improved vascularization compared to the material alone, with a corresponding upregulation of VEGF-C and bFGF [63]. Similarly, when combined with MSCs, this approach was shown to improve vascularization within bone defects of rhesus monkeys, further improved with a fascia flap surrounding the CaP [64] (Figure 2iii). In a medial thigh rat model using a BCP, the importance of both the intrinsic and extrinsic angiogenesis was demonstrated, where approximately $80 \%$ of the vessels were connected to the arteriovenous bundle after 2 weeks, increasing to $97 \%$ at 8 weeks [65]. While this approach is relevant and accessible, the relationship between vascularization, CaP degradation, and bone formation has not been well studied. In an intramuscular ectopic bone model in dogs, the higher flow rate of the arteriovenous bundle was shown to increase the sprouting of vessels over a ligated vascular bundle. However, this condition also corresponded with an increased $\mathrm{CaP}$ degradation rate, which resulted in less bone formation at 6 months than in the group with the ligated bundle [66]. Overall, surgical revascularization is an option that can be readily incorporated with $\mathrm{CaP}$ use. As a caveat to the benefits, the rate of failure due to thrombosis or other causes can be an issue [67]. In addition, further studies will be necessary to evaluate surgical strategies in comparison with biological or synthetic approaches.

Mechanical stimulation is another interesting in situ strategy for angiogenesis during bone healing. Indeed, a well-known aspect of healing is the response to mechanical stability and forces on bone formation. Wolff's law proposes that bone adapts and remodels according to mechanical stimuli, with osteocytes being mainly responsible for sensing external loads. Mechanotaxis - cell migration induced by changes in the mechanical environment - is well known to play a role in EC behavior via internal or external interactions via cytoskeletal protein filaments [68]. Vessels form preferentially from regions of higher deformation in vitro [69], although VEGF signaling was also reported to not be affected by either cyclic strains in vitro [70] or instability in a mouse tibial bone-fracture model [71]. This suggests the role of other mechanisms, such as those from angiopoietins that mediate endothelial cell-cell interactions, PDGF-bb involved in VSMC chemotaxis [70], and regulators such as Rho GTPases that can influence cell contraction [72]. In an unvalidated computational model simulating CaP use and cyclic strain, no difference in angiogenesis was calculated between $0.5 \%$ to $1 \%$ strain, while changes were confirmed in the osteoblast, chondrocyte, and fibroblast ratios, as well as increasing tissue stiffness over time [25]. Overall, while the mechanical aspects of osteogenesis and angiogenesis are very relevant to bone healing, there is a lack of information about whether externally applied mechanical stimulation within a CaP-augmented bone-healing site promotes vessel formation.

Other modes of stimulation, such as ultrasound or electromagnetic fields, have shown positive results for bone regeneration. Low-intensity pulsed ultrasound (LIPUS) has demonstrated an improved time to osteoporotic callus bridging in femoral fractures in rats, with increased VEGF expression [73]. When used with a $\beta$-TCP, the combination with LIPUS and MSCs improved spinal fusion in rabbits [74], indicating the benefits of combined approaches. With similar results, electrical stimulation and the generation of an electromagnetic field across a defect site have 
been shown to improve CaP osseointegration in the femoral cortical bone of rabbits [75]. In terms of angiogenesis, electromagnetic stimulation was shown to increase EC proliferation and tube-like formation. This positive effect on angiogenesis was even suggested to be the driver of the positive effects found within bone-healing studies, as opposed to having a direct effect on osteogenesis [76], although there is a lack of recent studies confirming this. Both ultrasound and electromagnetic stimulation have historically positive results for bone healing, even in combination with $\mathrm{CaPs}$, as the above studies demonstrate. While combination strategies have been understudied, there appears to be no reason for any detrimental effect on angiogenesis when combining stimulation with CaPs.

\section{Conclusions and Future Outlook}

$\mathrm{CaPs}$ in themselves seem to have the potential to influence angiogenesis. Nonetheless, current data are limited to explain the extent of this influence, and how it compares to other stimulatory and inhibitory mechanisms. Regardless, the positive results from various strategies combined with CaPs (Figure 3) demonstrate clear potential and call for further research and improvements (see Outstanding Questions). Implant design, proangiogenic factor delivery, and prevascularization are the major directions evolving to address the issue of vasculature. For these, many challenges still need to be overcome. The inclusion of ions, molecules, or growth factors requires better temporal control of their release, which could allow greater control of the bone-healing mechanisms. The benefits of completely synthetic approaches are that they do not rely on exogenous or recombinant factors and do not require the harvesting or in vitro culturing of cells. However, recurring concerns about the toxicity of additives need to be further considered. In addition, immunomodulation, such as macrophage polarization, may prove worthwhile to explore [77], although further research will be necessary to confirm the direct connection between CaPs, immunomodulation, and revascularization. Lastly, in vitro prevascularization needs to address issues related to associated cell death and failures in connecting to the host system [48,78]. Taken together, CaPs have clear benefits for osteogenic processes and have shown positive results for bone healing in general. To advance this material further, concerted efforts must be devoted to study the effects of $\mathrm{CaP}$ materials, and almost all bone-graft substitutes, on angiogenic responses.

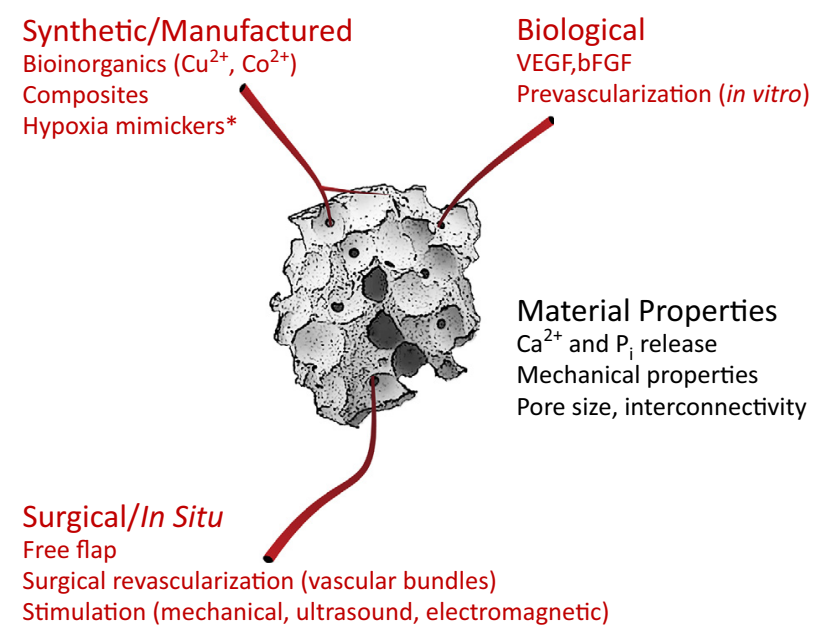

Trends in Biotechnology

Figure 3. Multiple Options To Address Angiogenesis Using CaP Bone-Graft Substitutes. Vascularization (red vessels) within $\mathrm{CaP}$ (grey granule) can be stimulated by modifications to the material itself (black text) or by complementary approaches (red text). *Hypoxia mimickers can also be biological.

\section{Outstanding Questions}

Is optimizing the physicochemical, structural, and mechanical properties of CaPs a sufficiently strong tool to stimulate angiogenesis without the need for additives?

Will inorganic compounds or small molecules be sufficient to replace biological approaches in stimulating angiogenesis in $\mathrm{CaPs}$ ?

What are the underlying mechanisms behind the effects of individual properties of (functionalized) CaPs on angiogenesis and bone formation, and how can they be better employed to optimize the clinical performance of $\mathrm{CaPs}$ ?

How can in vitro models encompass more aspects of bone healing, including angiogenesis, to accelerate $\mathrm{CaP}$ bone-graft substitute research?

Will the strategies to address both bone formation and angiogenesis/vascularization in CaPs lead to synthetic bone graft substitutes that can fully replace autografts? 


\section{Acknowledgments}

The authors acknowledge The Netherlands Science Organization (NWO) for financial support (Aspasia premium number 015.008.039). This research has been in part made possible with the support of the Dutch Province of Limburg.

\section{References}

1. Albrektsson, T. and Johansson, C. (2001) Osteoinduction, osteoconduction and osseointegration. Eur. Spine J. 10 (Suppl. 2), 96101

2. Giannoudis, P.V. et al. (2007) Fracture healing: the diamond concept. Injury 38 (Suppl. 4), S3-S6

3. Yuan, H. et al. (2010) Osteoinductive ceramics as a synthetic alternative to autologous bone grafting. Proc. Natl. Acad. Sci. U.S.A. $107,13614-13619$

4. Habibovic, P. et al. (2008) Comparative in vivo study of six hydroxyapatite-based bone graft substitutes. J. Orthop. Res. 26, 1363-1370

5. Habibovic, P. and de Groot, K. (2007) Osteoinductive biomaterials - properties and relevance in bone repair. J. Tissue Eng. Regen. Med. 1, 25-32

6. Bose, S. and Tarafder, S. (2012) Calcium phosphate ceramic systems in growth factor and drug delivery for bone tissue engineering: a review. Acta Biomater. 8, 1401-1421

7. Giannoudis, P.V. et al. (2008) The diamond concept - open questions. Injury 39 (Suppl. 2), S5-S8

8. Malhotra, A. et al. (2013) Can platelet-rich plasma (PRP) improve bone healing?. A comparison between the theory and experimental outcomes. Arch. Orthop. Trauma Surg. 133, 153-165

9. Schmidt-Bleek, K. et al. (2012) Inflammatory phase of bone healing initiates the regenerative healing cascade. Cell Tissue Res. 347, 567-573

10. Kanczler, J.M. and Oreffo, R.O. (2008) Osteogenesis and angiogenesis: the potential for engineering bone. Eur. Cell Mater. 15, 100-114

11. Larsen, M. et al. (2011) Living bone allotransplants survive by surgical angiogenesis alone: development of a novel method of composite tissue allotransplantation. J. Bone Joint Surg. Am. 93, 261-273

12. Gotz, W. et al. (2012) Coupling of osteogenesis and angiogenesis in bone substitute healing - a brief overview. Ann. Anat. 194, 171173

13. Munaron, L. and Pla, A.F. (2009) Endothelial calcium machinery and angiogenesis: understanding physiology to interfere with pathology. Curr. Med. Chem. 16, 4691-4703

14. Clapham, D.E. (2007) Calcium signaling. Cell 131, 1047-1058

15. Kohn, E.C. et al. (1995) Angiogenesis: role of calcium-mediated signal transduction. Proc. Natl. Acad. Sci. U.S.A. 92, 1307-1311

16. Sapir-Koren, R. and Livshits, G. (2011) Bone mineralization and regulation of phosphate homeostasis. IBMS BoneKEy 8, 286-300

17. Fukumoto, S. (2014) Phosphate metabolism and vitamin D. BoneKEy Rep. 3, 497

18. Habibovic, P. et al. (2010) Collagen biomineralization in vivo by sustained release of inorganic phosphate ions. Adv. Mater. 22 1858-1862

19. Shanahan, C.M. et al. (2011) Arterial calcification in chronic kidney disease: key roles for calcium and phosphate. Circ. Res. 109, 697-711

20. Nigwekar, S.U. et al. (2014) Vitamin D and chronic kidney diseasemineral bone disease (CKD-MBD). BoneKEy Rep. 3, 498

21. Di Marco, G.S. et al. (2013) High phosphate directly affects endothelial function by downregulating annexin II. Kidney Int. 83, 213222

22. Di Marco, G.S. et al. (2008) Increased inorganic phosphate induces human endothelial cell apoptosis in vitro. Am. J. Physiol. Renal Physiol. 294, F1381-F1387

23. Barrere, F. et al. (2006) Bone regeneration: molecular and cellular interactions with calcium phosphate ceramics. Int. J. Nanomedicine 1, 317-332

24. Jufri, N.F. et al. (2015) Mechanical stretch: physiological and pathological implications for human vascular endothelial cells. Vasc. Cell 7, 8
25. Sandino, C. et al. (2010) Simulation of angiogenesis and cel differentiation in a CaP scaffold subjected to compressive strains using a lattice modeling approach. Biomaterials 31, 2446-2452

6. Klenke, F.M. et al. (2008) Impact of pore size on the vascularization and osseointegration of ceramic bone substitutes in vivo. J. Biomed. Mater. Res. A 85, 777-786

27. Bai, F. et al. (2011) The effect of pore size on tissue ingrowth and neovascularization in porous bioceramics of controlled architecture in vivo. Biomed. Mater. 6, 015007

28. Bai, F. et al. (2010) The correlation between the internal structure and vascularization of controllable porous bioceramic materials in vivo: a quantitative study. Tissue Eng. Part A 16, 3791-3803

29. Xiao, X. et al. (2015) The promotion of angiogenesis induced by three-dimensional porous beta-tricalcium phosphate scaffold with different interconnection sizes via activation of PI3K/Akt pathways. Sci. Rep. 5, 9409

30. Chen, Y. et al. (2015) Enhanced effect of $\beta$-tricalcium phosphate phase on neovascularization of porous calcium phosphate ceramics: in vitro and in vivo evidence. Acta Biomater. 11, 435-448

31. Hutmacher, D.W. et al. (2007) State of the art and future directions of scaffold-based bone engineering from a biomaterials perspective. J. Tissue Eng. Regen. Med. 1, 245-260

32. Amini, A.R. et al. (2012) Bone tissue engineering: recent advances and challenges. Crit. Rev. Biomed. Eng. 40, 363-408

33. Malhotra, A. et al. (2014) Platelet-rich plasma and bone defect healing. Tissue Eng. Part A 20, 2614-2633

34. Xiu, J. et al. (2014) Different angiogenic abilities of self-setting calcium phosphate cement scaffolds consisting of different proportions of fibrin glue. Biomed. Res. Int. 2014, 785146

35. Kang, W. et al. (2015) Fluorescence-based retention assays reveals sustained release of vascular endothelial growth factor from bone grafts. J. Biomed. Mater. Res. A 104, 283-290

36. Burdyga, T. and Borysova, L. (2014) Calcium signalling in pericytes. J. Vasc. Res. 51, 190-199

37. Wernike, E. et al. (2010) VEGF incorporated into calcium phosphate ceramics promotes vascularisation and bone formation in vivo. Eur. Cell. Mater. 19, 30-40

38. Moon, K.S. et al. (2015) The effect of covalently Immobilized FGF-2 on biphasic calcium phosphate bone substitute on enhanced biological compatibility and activity. Biomed. Res. Int. 2015, 742192

39. Minamide, A. et al. (2007) The effects of bone morphogenetic protein and basic fibroblast growth factor on cultured mesenchymal stem cells for spine fusion. Spine 32, 1067-1071

40. Sukul, M. et al. (2015) Effect of local sustainable release of BMP2 VEGF from nano-cellulose loaded in sponge biphasic calcium phosphate on bone regeneration. Tissue Eng. Part A 21, 1822 1836

41. Rouwkema, J. et al. (2006) Endothelial cells assemble into a 3 dimensional prevascular network in a bone tissue engineering construct. Tissue Eng. 12, 2685-2693

42. Portalska, K.J. et al. (2014) Boosting angiogenesis and functiona vascularization in injectable dextran-hyaluronic acid hydrogels by endothelial-like mesenchymal stromal cells. Tissue Eng. Part A 20, 819-829

43. Portalska, K.J. et al. (2016) Collagen modules for in situ delivery of mesenchymal stromal cell-derived endothelial cells for improved angiogenesis. J. Tissue Eng. Regen. Med. 10 (5), 363-373

44. Unger, R.E. et al. (2007) Tissue-like self-assembly in cocultures of endothelial cells and osteoblasts and the formation of microcapillary-like structures on three-dimensional porous biomaterials. Biomaterials 28, 3965-3976

45. Thein-Han, W. and XU, H.H.K. (2013) Prevascularization of a gasfoaming macroporous calcium phosphate cement scaffold via coculture of human umbilical vein endothelial cells and osteoblasts. Tissue Eng. Part A 19, 1675-1685 
46. Chen, W. et al. (2014) Prevascularization of biofunctional calcium phosphate cement for dental and craniofacial repairs. Dent. Mater. 30, 535-544

47. Rivron, N.C. et al. (2012) Sonic Hedgehog-activated engineered blood vessels enhance bone tissue formation. Proc. Natl. Acad. Sci. U.S.A. 109, 4413-4418

48. Stegen, S. et al. (2015) Bringing new life to damaged bone: the importance of angiogenesis in bone repair and regeneration. Bone 70, 19-27

49. Spadaro, J. et al. (1970) The distribution of trace metal ions in bone and tendon. Calcif. Tissue Res. 6, 49-54

50. Habibovic, P. and Barralet, J.E. (2011) Bioinorganics and biomaterials: bone repair. Acta Biomater. 7, 3013-3026

51. Fu, W.L. et al. (2015) Coculture of peripheral blood-derived mesenchymal stem cells and endothelial progenitor cells on strontiumdoped calcium polyphosphate scaffolds to generate vascularized engineered bone. Tissue Eng. Part A 21, 948-959

52. Barralet, J. et al. (2009) Angiogenesis in calcium phosphate scaffolds by inorganic copper ion release. Tissue Eng. Part A 15, 1601-1609

53. Bose, S. et al. (2013) Understanding of dopant-induced osteogenesis and angiogenesis in calcium phosphate ceramics. Trends Biotechnol. 31, 594-605

54. Yang, L. et al. (2010) The effects of inorganic additives to calcium phosphate on in vitro behavior of osteoblasts and osteoclasts. Biomaterials 31, 2976-2989

55. Zeinab Tahmasebi, B. et al. (2016) Combinatorial incorporation of fluoride and cobalt ions into calcium phosphates to stimulate osteogenesis and angiogenesis. Biomed. Mater. 11, 015020

56. Fuchs, S. et al. (2010) Influence of polymer content in Ca-deficient hydroxyapatite-polycaprolactone nanocomposites on the formation of microvessel-like structures. Acta Biomater. 6, 3169-3177

57. Oliveira, H. et al. (2016) The proangiogenic potential of a novel calcium releasing biomaterial: Impact on cell recruitment. Acta Biomater. 29, 435-445

58. Doorn, J. et al. (2013) A small molecule approach to engineering vascularized tissue. Biomaterials 34, 3053-3063

59. Wu, C. et al. (2012) Hypoxia-mimicking mesoporous bioactive glass scaffolds with controllable cobalt ion release for bone tissue engineering. Biomaterials 33, 2076-2085

60. Simon, M.C. and Keith, B. (2008) The role of oxygen availability in embryonic development and stem cell function. Nat. Rev. Mol. Cell Biol. 9, 285-296

61. Harry, L.E. et al. (2008) Comparison of the healing of open tibial fractures covered with either muscle or fasciocutaneous tissue in a murine model. J. Orthop. Res. 26, 1238-1244

62. Henkel, J. et al. (2013) Bone regeneration based on tissue engineering conceptions-a 21st century perspective. Bone Res. 3, 216-248

63. Yang, P. et al. (2013) Development of a new pre-vascularized tissue-engineered construct using pre-differentiated rADSCs, arteriovenous vascular bundle and porous nano-hydroxyapatide-polyamide 66 scaffold. BMC Musculoskelet. Disord. 14, 1-14

64. Fan, H. et al. (2014) Efficacy of prevascularization for segmental bone defect repair using $\beta$-tricalcium phosphate scaffold in rhesus monkey. Biomaterials 35, 7407-7415

65. Arkudas, A. et al. (2012) Combination of extrinsic and intrinsic pathways significantly accelerates axial vascularization of bioartificial tissues. Plast. Reconstr. Surg. 129, 55e-65e

66. Wu, X. et al. (2015) The effects of different vascular carrier patterns on the angiogenesis and osteogenesis of BMSC-TCP-based tissueengineered bone in beagle dogs. J. Tissue Eng. Regen. Med. Published online August 7, 2015. http://dx.doi.org/10.1002/term.2076

67. Boos, A.M. et al. (2013) Engineering axially vascularized bone in the sheep arteriovenous-loop model. J. Tissue Eng. Regen. Med. 7, 654-664

68. Ingber, D.E. and Folkman, J. (1989) Mechanochemical switching between growth and differentiation during fibroblast growth factor- stimulated angiogenesis in vitro: role of extracellular matrix. J. Cell Biol. 109, 317-330

69. Rivron, N.C. et al. (2012) Tissue deformation spatially modulates VEGF signaling and angiogenesis. Proc. Natl. Acad. Sci. U.S.A 109, 6886-6891

70. Yung, Y.C. et al. (2009) Cyclic tensile strain triggers a sequence of autocrine and paracrine signaling to regulate angiogenic sprouting in human vascular cells. Proc. Natl. Acad. Sci. U.S.A. 106, 15279 15284

71. Lu, C. et al. (2011) Mechanical stability affects angiogenesis during early fracture healing. J. Orthop. Trauma 25, 494-499

72. Etienne-Manneville, S. and Hall, A. (2002) Rho GTPases in cell biology. Nature 420, 629-635

73. Cheung, W-H. et al. (2011) Low-intensity pulsed ultrasound accelerated callus formation, angiogenesis and callus remodeling in osteoporotic fracture healing. Ultrasound Med. Biol. 37, 231-238

74. Hui, C.F.F. et al. (2011) Low-ontensity pulsed ultrasound enhances posterior spinal fusion implanted with mesenchymal stem cells-calcium phosphate composite without bone grafting. Spine 36, 1010-1016

75. Fini, M. et al. (2006) Histomorphometric and mechanical analysis of the hydroxyapatite-bone interface after electromagnetic stimulation-an experimental study in rabbits. J. Bone Joint Surg. Br. 88, 123-128

76. Tepper, O.M. et al. (2004) Electromagnetic fields increase in vitro and in vivo angiogenesis through endothelial release of FGF-2. FASEB J. 18, 1231

77. Velard, F. et al. (2013) Inflammatory cell response to calcium phosphate biomaterial particles: an overview. Acta Biomater. 9 4956-4963

78. Jain, R.K. et al. (2005) Engineering vascularized tissue. Nat. Biotechnol. 23, 821-823

79. Potente, M. et al. (2011) Basic and therapeutic aspects of angiogenesis. Cell 146, 873-887

80. Kusumbe, A.P. et al. (2014) Coupling of angiogenesis and osteogenesis by a specific vessel subtype in bone. Nature 507, 323328

81. le Noble, F. and le Noble, J. (2014) Bone biology: vessels of rejuvenation. Nature 507, 313-314

82. Carmeliet, P. (2005) Angiogenesis in life, disease and medicine. Nature 438, 932-936

83. Caplan, A.I. and Correa, D. (2011) PDGF in bone formation and regeneration: new insights into a novel mechanism involving MSCs. J. Orthop. Res. 29, 1795-1803

84. Khakoo, A.Y. and Finkel, T. (2005) Endothelial progenitor cells. Annu. Rev. Med. 56, 79-101

85. Galis, Z.S. and Khatri, J.J. (2002) Matrix metalloproteinases in vascular remodeling and atherogenesis - the good, the bad, and the ugly. Circ. Res. 90, 251-262

86. Folkman, J. and Shing, Y. (1992) Angiogenesis. J. Biol. Chem 267 , 10931-10934

87. Carmeliet, P. and Jain, R.K. (2000) Angiogenesis in cancer and other diseases. Nature 407, 249-257

88. Henno, S. et al. (2003) Characterisation and quantification of angiogenesis in $\beta$-tricalcium phosphate implants by immunohistochemistry and transmission electron microscopy. Biomaterials 24, 3173-318

89. Hadijpanayi, E. and Schilling, A.F. (2013) Hypoxia-based strategies for angiogenic induction: The dawn of a new era for ischemia therapy and tissue regeneration. Organogenesis 9, 261-272

90. Knighton, D.R. et al. (1983) Oxygen tension regulates the expression of angiogenesis factor by macrophages. Science 221, 1283 1285

91. Rouwkema, J. et al. (2008) Vascularization in tissue engineering Trends Biotechnol. 26, 434-441 